\title{
Structural Study of the Legionella pneumophila Dot/Icm T4SS Using Cryo-electron Microscopy
}

Clarissa Durie $^{1}$, Michael Sheedlo ${ }^{2}$, Jeong Min Chung ${ }^{1}$, Brenda Byrne ${ }^{3}$, Thomas Knight ${ }^{3}$, Michele Swanson $^{3}$, Dana Lacy ${ }^{2}$ and Melanie Ohi ${ }^{1}$

${ }^{1}$ University of Michigan, Ann Arbor, Michigan, United States, ${ }^{2}$ Vanderbilt University Medical Center, Nashville, Tennessee, United States, ${ }^{3}$ University of Michigan Medical School, Ann Arbor, Michigan, United States

Bacterial pathogens are a threat to global health and have evolved elaborate strategies to infect their hosts. One potent bacterial weapon in the war between host and pathogen is the Type IV secretion system (T4SS). In Gram negative bacteria these complexes can deliver: 1) effector proteins into eukaryotic cells, 2) DNA into other bacteria, or 3) toxins into bacterial neighbors. T4SSs are found in a wide variety of bacterial species including those that cause human disease, such as Legionella pneumophila, Helicobacter pylori, Bordetella pertussis, Brucella, Bartonella, and Coxiella. When inhaled, L. pneumophila replicates in alveolar macrophages and can cause a progressive and potentially fatal pneumonia, called Legionnaire's disease $(1,2)$. Infection begins when macrophages phagocytose the opportunistic pathogen which then co-opts the host cell phagosome to create a replicative niche (3). The Defect in Organelle Transport (Dot) T4SS is required for this process and essential for L. pneumophila pathogenesis. This complex secretes hundreds of bacterial proteins into the host cell that work to subvert host cellular machinery, inhibiting phagosome fusion with the lysosome and bacteria degradation.

T4SSs are challenging complexes to work with because they span the inner and outer membranes of Gramnegative bacteria and contain a minimum of 12 proteins. Studies in prototype systems, such as $E$. coliplasmids and A. tumefaciens show that T4SSs are generally organized into an outer membrane core complex (OMCC), an inner membrane complex (IMC), and in some species an extracellular pilus(4-6). Recent biochemical and in vivo cryo-electron tomography (cryo-ET) studies of L. pneumophila and $H$. pylori T4SSs show these complexes have distinctive structural features compared to previously characterized T4SSs (7-12). Differences include: 1) the OMCCs contain at least five proteins instead of three, 2) the OMCCs are larger and more intricately organized than those in X. citri and conjugation systems, and 3) the T4SSs have periplasmic sub-complexes not seen in other bacteria. Since $L$. pneumophila and $H$. pylori T4SSs secrete proteins rather than DNA, we predict that these structural differences are essential for function and will provide molecular insight into the mechanism of T4SS substrate specificity.

The L. pneumophila T4SS is composed of $\sim 26$ components and low resolution ( 20-40 ̊) cryo-electron tomography studies show that this T4SS shares some architectural characteristics with the $H$. pylori T4SS $(8,9,11-16)$. However, the lack of detailed structural information limits our mechanistic understanding of how the L. pneumophila T4SS functions and contributes to pathogenesis. I have biochemically purified the L.pneumophila T4SS core complex and visualized it by cryo-EM. From this analysis it is clear that while similarities between the Cag and Dot T4SSs exist, the L. pneumophila T4SS is structurally distinct from other T4SS complexes. 


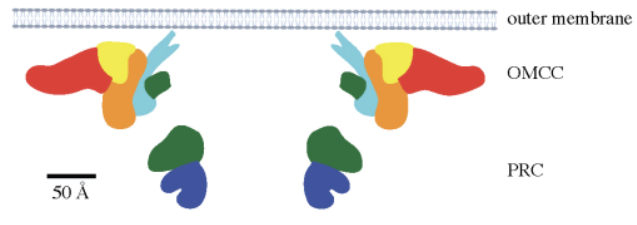

Figure 1. Figure 1. Cartoon schematic showing the organization of the L. pneumophila Dot T4SS outer membrane core complex (OMCC) and periplasmic ring complex (PRC) in relation to the outer membrane. The central axial slice view is shown. Different protein core components are shown in different colors.

\begin{tabular}{|c|ccc|}
\hline Identified Proteins & Prep 1 & $\begin{array}{c}\text { Spectral Counts } \\
\text { Prep 2 }\end{array}$ & Prep 3 \\
\hline DotG & 112 & 114 & 195 \\
DotF & 94 & 69 & 101 \\
DotA & 38 & 65 & 60 \\
DotO & 37 & 38 & 47 \\
DotH & 28 & 19 & 28 \\
IcmF & 15 & 18 & 36 \\
IcmX (IcmY) & 19 & 13 & 28 \\
DotL & 10 & 26 & 20 \\
DotC & 9 & 11 & 16 \\
DotD & 11 & 9 & 14 \\
DotB & 16 & 11 & 7 \\
DotM & 2 & 14 & 3 \\
DotK & 2 & 6 & 10 \\
IsmW & 5 & 7 & 5 \\
DotN & 2 & 6 & 4 \\
DotI & 1 & 3 & 5 \\
IcmS & & 4 & 1 \\
\hline \multicolumn{2}{|c}{}
\end{tabular}

Figure 2. Table 1. T4SS components identified by mass spectrometry. Spectral counts of all Dot/Icm proteins are shown across three biological replicates of complex isolation.

References

1. Molofsky AB, Swanson MS. 2004. Mol Microbiol 53: 29-40

2. Swanson MS, Hammer BK. 2000. Annu Rev Microbiol 54: 567-613

3. Nora T, Lomma M, Gomez-Valero L, Buchrieser C. 2009. Future Microbiol 4: 691-701

4. Christie PJ, Cascales E. 2005. Mol Membr Biol 22: 51-61

5. Grohmann E, Christie PJ, Waksman G, Backert S. 2018. Mol Microbiol 107: 455-71

6. Waksman G. 2019. EMBO Rep 20

7. Frick-Cheng AE, Pyburn TM, Voss BJ, McDonald WH, Ohi MD, Cover TL. 2016. MBio 7: e02001-15

8. Chang YW, Shaffer CL, Rettberg LA, Ghosal D, Jensen GJ. 2018. Cell Rep 23: 673-81

9. Ghosal D, Chang YW, Jeong KC, Vogel JP, Jensen GJ. 2017. EMBO Rep 18: 726-32

10. Chung JM, Sheedlo MJ, Campbell AM, Sawhney N, Frick-Cheng AE, et al. 2019. Elife 8

11. Chetrit D, Hu B, Christie PJ, Roy CR, Liu J. 2018. Nat Microbiol 3: 678-86

12. Park D, Chetrit D, Hu B, Roy CR, Liu J. 2020. mBio 11

13. Segal G, Purcell M, Shuman HA. 1998. Proc Natl Acad Sci U S A 95: 1669-74

14. Segal G, Shuman HA. 1999. Infect Immun 67: 2117-24

15. Vogel JP, Andrews HL, Wong SK, Isberg RR. 1998. Science 279: 873-6

16. Hu B, Khara P, Song L, Lin AS, Frick-Cheng AE, et al. 2019. mBio 10 